\title{
Gratitude and Life Satisfaction: Mediating Role of Perceived Stress
}

\author{
Murat Yildirim $^{1} \&$ Zainab Shalal Alanazi ${ }^{1,2}$ \\ ${ }^{1}$ Department of Neuroscience, Psychology and Behaviour, George Davies Centre, University of Leicester, \\ Leicester, United Kingdom \\ ${ }^{2}$ Department of Psychology, Shaqra University, Muzahimiyah, Saudi Arabia.
}

Correspondence: Murat Yildirim, Department of Neuroscience, Psychology and Behaviour, University of Leicester. George Davies Centre, University Road, Leicester, LE1 9HN, United Kingdom. E-mail: my109@1e.ac.uk

Received: June 23, 2018

doi:10.5539/ijps.v10n3p21
Accepted: July 9, 2018

Online Published: July 16, 2018

URL: https://doi.org/10.5539/ijps.v10n3p21

\begin{abstract}
The relationships between gratitude, satisfaction with life, and stress have been widely examined in different cultures. However, empirical research on these variables is scant in Saudi Arabia. The aim of this study was to investigate the mediation effect of stress in the relationship between gratitude and life satisfaction in an understudied population of Arabic student sample. Participants were 141 Arabic-speaking undergraduate students $(18$ males and 123 females; mean age $=23.8$ years, $S D=4.23)$ and completed self-report measures of gratitude, satisfaction with life, and stress. As expected, regression analysis showed that gratitude positively predicted satisfaction with life, while stress negatively predicted satisfaction with life. Mediation analysis showed that stress fully mediate the relationship between gratitude and life satisfaction. Higher levels of gratitude positively predicted higher levels of satisfaction with life though the decreased stress. The emerging results have important implications to research and practice regarding understanding the mechanism underlying gratitude, satisfaction with life, and stress in the context of a non-western country.
\end{abstract}

Keywords: gratitude, satisfaction with life, perceived stress, mediation, Saudi Arabia

\section{Introduction}

The concept of subjective well-being (SWB) is conceptualized as including three important elements: (a) one's cognitive assessment of his or her life in general and specific aspects of life, (b) the presence of positive affect, and (c) the absence of negative affect. The former refers to cognitive component of SWB, while the latter two refer to affective components of SWB (Diener, 1984, 2000; Diener \& Suh, 1997). Individuals high in SWB is described as experiencing low negative affect, high positive affect, and high satisfaction with life (Diener \& Lucas, 1999). Research showed that three elements of SWB (positive affect, negative affect and satisfaction with life) are related with one another yet distinct from each other (Diener et al., 1999; Lucas, Diener \& Suh, 1996). As seen, satisfaction with life is one of the prominent components of SWB. Satisfaction with life is relatively a more stable characteristic than affective components and it has extensive benefits to the psychological health and quality of life of individuals. Satisfaction with life allows individuals to perform long-term life evaluations based on their own sets of criteria (Eid \& Diener, 2004; Pavot \& Diener, 1993).

Numerous studies have been conducted to determine the correlates and predictors of satisfaction with life across cultures. Studies have shown that there is a wide range of factors that influence satisfaction with life or SWB. Some of these factors are personality, social support, health, and individualism vs collectivism (Diener \& Diener, 1995; Diener \& Lucas, 1999; Diener, Oishi \& Lucas, 2003). Studies also suggest that higher satisfaction with life is related with higher resilience, self-esteem (Lee, Kim \& Wachholtz, 2016; Martínez-Martí \& Ruch, 2017), presence of partnership, and income while lower satisfaction with life is related with higher stress (Lee, Kim \& Wachholtz, 2016), anxiety, depression, unemployment (Beutel et al., 2010), fear of happiness (Yildirim \& Aziz, 2017), and externality of happiness (Yildirim, Barmanpek, \& Farag, 2018a). Satisfaction with life is associated with gratitude and social support (Kong, Ding \& Zhao, 2015; Wood, Joseph \& Maltby, 2008a), optimism (Extremera, Durán \& Rey, 2009), social relationships (Darling et al., 2007), and health seeking and health-promoting behaviours (Grant, Wardle \& Steptoe, 2009). Furthermore, studies revealed that perceived stress and availability of coping recourses significantly predict life satisfaction (Hamarat et al., 2001; Simons et 
al., 2002).

Gratitude is an important positive concept that has drawn an intense interest with the advent of positive psychology. The concept of gratitude has been characterised as a stable inclination that refers to "a general tendency to recognize and respond with grateful emotion to the roles of other people's benevolence in the positive experiences and outcomes that one obtains" (McCullough, Emmons, \& Tsang, 2002). There are variety of benefits of having feelings of gratitude across different life domains such as physical health, mental health and social well-being (Lambert et al., 2009). Grateful individuals tend to be more orientated toward social interaction by showing prosocial behaviours, in that they are more empathic, forgiving, helpful, and supportive to others alongside seeking for less materialistic achievements (McCullough et al., 2002). Being grateful for the things in life also allow individuals to develop better social relationships, experience greater satisfaction and enjoyment with life, and cope with stressful situations in their life (Lyubomirsky, Sheldon \& Schkade, 2005). Furthermore, individuals are reporting higher feelings of gratitude report higher levels of positive feelings, life satisfaction, and spiritual well-being and lower level of negative feelings including depression, anxiety, envy (McCullough et al., 2002), and perceived stress (Lee et al. 2018).

Empirical evidence showed that gratitude is positively associated with autonomy, environment mastery, personal growth, positive relationships with others, purpose in life, self-acceptance (Wood, Joseph, \& Maltby, 2009), satisfaction with life (Emmons \& McCullough, 2003; Lavy \& Littman-Ovadia, 2011; McCullough et al., 2002; Wood et al., 2008a), and greater positive appraisal in life (Emmons \& McCullough, 2003). Gratitude is also correlated with both the domains and facets of the Big Five model of personality (Wood et al., 2008a; 2009). Gratitude uniquely contributes to both satisfaction with life and psychological well-being after controlling for both the domains and facets of the five-factor model of personality (Wood et al., 2008a; 2009). Additionally, recent longitudinal studies on the associations of gratitude to psychological well-being in the context of chronic illness revealed that gratitude uniquely predicts lower depression six months apart (Sirois \& Wood, 2017). Studies using cross-lagged panel design on healthy individuals also revealed similar results. In a study, Wood et al. (2008b) investigated direct, reverse, and reciprocal relationships of gratitude in the development of social support, stress, and depression. The results indicated that gratitude directly resulted in greater levels of perceived social support, and lower levels of stress and depression. Their results also showed that gratitude uniquely influenced the above-mentioned variables beyond the effect Big Five model of personality. However, Wood et al. failed to provide evidence for reverse of the reciprocal relationships among the variables.

\subsection{Mediating Role of Perceived Stress}

The construct of stress has received immense attentions due to its impact on human functioning. One of the most prominent theories within the psychological literature on stress is Transactional Model of Stress (Lazarus \& Folkman, 1984). According to this theory, stress is a dynamic interaction between an individual and his/her surroundings. Within the theory, stress is seen as a product of imbalance between the individual's perception of the surrounding and his personal abilities and resources to deal with the stressors. Stress is also defined as a state of threatened homeostasis resulted in by stressors and is neutralized by a tangled repertoire of physiological and behavioural responses seeking for maintaining or re-establishing the optimal body equilibrium (Tsigos et al., 2016).

Findings revealed that perceived stress is a significant predictor of life satisfaction (Hamarat et al., 2001). Perceived stress was found positively linked with increased symptoms of minor psychiatric disorders and postnatal depression (Chaaya et al., 2010). Atanes et al. (2015) indicated that individuals who reported higher levels of perceived stress were more likely to report lower levels of satisfaction with life, positive affect, and mindfulness, and higher levels of negative affect. Perceived stress was also found negatively associated with perceived health (The et al., 2015). That is, those who report higher levels of perceived stress tend to have lower levels of health perception, mental health problems, emotional problems, social functioning, physical functioning, bodily pain, physical health problems, and energy. Finding from recent experimental and longitudinal studies suggested that perceived stress is an important determinant of SWB (Praharso, Tear \& Cruwys, 2017). Furthermore, many empirical studies have shown that stress is associated with gratitude (e.g., Lin, 2015; Wood et al., 2008b). For example, in a study, Lee et al. (2018) found that gratitude negatively predicted perceived stress among male firefighters in Korea after controlling for gratitude-related factors such as religiousness, personality variables, anxiety, and depression. They suggest that gratitude functions as a unique protective factor against precevied stress. Thus, perceived stress is likely to act as a mediator in the relationship between gratitude and satisfaction with life. 


\subsection{Present Research}

Despite the large volume of research on gratitude, satisfaction with life, and stress in different cultures, particularly in western cultures, empirical research on these variables is scant in Saudi Arabia. It is important to provide empirical support for the role of gratitude in predicting satisfaction with life, particularly through other psychological variables such as stress. This would help us to understand the underlying mechanism of the relationship between gratitude and other variables. The present study investigated the mediator effect of stress in the relationship between gratitude and satisfaction with life in an Arabic-speaking population. Investigating the role of gratitude on satisfaction with life via stress would contribute to extant literature on the relevant constructs. Hence, this study particularly aimed to examine the impact of stress in the relationships between gratitude and life satisfaction. A model was proposed by the researchers to explore these relationships. Theoretical conceptualization of these relationships is presented in Figure 1. Based on this conceptualization, it was hypothesised that gratitude would positively predict life satisfaction. It was also hypothesised that perceived stress would predict life satisfaction. Furthermore, it was hypothesised that perceived stress would fully mediate the relationships between gratitude and life satisfaction. That is, a high level of gratitude would contribute to lower stress and that in turn would contribute to higher satisfaction with life.

\section{Method}

\subsection{Participants}

A total of 141 Arabic-speaking undergraduate students were recruited for the study. $87.2 \%$ of the participants were females and $12.8 \%$ were males. Participants ages ranged from 18 to 47 , with the average age being 23.8 $(S D=4.23)$. Of the participants, $78.7 \%$ were single, while $21.3 \%$ were married. Participants self-identified themselves as having different socioeconomic backgrounds (Low $=1.4 \%$, Medium $=44 \%$, High $=53.2 \%$, Very high $=1.4 \%$ ). Volunteer students only took part in the study.

\subsection{Measures}

2.2.1 Gratitude Questionnaire (GQ-6; McCullough et al. 2002). The GQ-6 is developed to measure dispositional aspect of gratitude and comprises of 6 items. Each item is answered on a 7-point scale ranging from 1 (strongly disagree) to 7 (strongly agree). A sample item is "I have so much in life to be thankful for." The scale scores are the sum of items after reversing relevant items. Higher scores on the scale refer to higher level of gratitude. For the purpose of this study, forward-backward translation procedure was used to translate the scale into Arabic language. In the original article, the GQ-6 provided excellent psychometric properties with a robust one-factor solution and high internal consistency. Cronbach's alpha for the scale in this study was .61.

2.2.2 Satisfaction with Life Scale (SWLS; Diener, Emmons, Larsen, \& Griffin, 1985). The SWLS is widely used to measure life satisfaction. The scale was developed to assess individuals' general satisfaction with life. The SWLS is a unidimensional scale that includes 5 items with high excellent psychometric properties (Diener et al., 1985). Each item is answered on a 7-point scale ranging from 1 (strongly disagree) to 7 (strongly agree). A sample item is "In most ways my life is close to my ideal." The scale scores are the sum of the items. Higher scores on the scale indicate greater satisfaction with life. The scale was adapted into Arabic language by Abdel-Khalek (2008). Cronbach's alpha for the scale in this study was .53.

2.2.3 Perceived Stress Scale (PSS; Cohen, Kamarck, and Mermelstein, 1983). The PSS is a global measure of perceived stress. The scale is developed to assess how situations in one's life are perceived as stressful in terms of being unpredictable, uncontrollable, and overloaded. The scale includes 10 items and each item is answered on a 5-point scale ranging from 0 (never) to 4 (very often). A sample item is "In the last month, how often have you been upset because of something that happened unexpectedly?" The scale scores are the sum of the items after reversing four positively stated items. Higher scores indicate greater perceived stress level in the last month. The scale was adapted into Arabic language by Chaaya et al. (2010). Cronbach's alpha for the scale in this study was .66

\subsection{Procedure}

The questionnaires were delivered to the university students in designated IT laboratories. Data was collected via an on-line questionnaire promoted by means of a secure software. Participants were asked to sit comfortably during completing the questionnaires. Participants were provided with a link to click to access the study. Before beginning the package of questionnaire, participants were asked to read and sign an informed consent form. The form included information regarding the purpose of the study, assurance of anonymity and confidentiality of the responses, storing, and disposing of the data. The form also consisted of information regarding the participants' right to withdraw from the study both during and after the participation (e.g., withdraw from the study at any 
time point without giving any reasons). All participants were volunteers and did not receive any type of momentary award for contributing to the study. The questionnaires were presented to the participants in the same order. Each participant completed the questionnaires individually. Competent assistants performed the administration of the above-mentioned questionnaires.

\subsection{Statistical Analysis}

Preliminary analyses were conducted to investigate whether the assumptions for the analysis were met. Skewness and kurtosis values were used to test normal distributions of the study variables. Using standardized Z scores, no univariate outliers were determined for any of the study variables. Multivariate outliers were tested using Mahalanobis distance and no multivariate outliers were identified. Multicollinearity issues were examined using standard linear regression. Variance inflation factor (range: 1.108 - 1.1.593) and tolerance (range: .628 - .902) did not reveal any multicollinearity issue. Pearson correlation coefficients were computed to explore the relationships among the variables. Regression analysis was used to estimate the direct effects of variables on satisfaction with life. Mediation analysis in regression was used to estimate indirect effect of stress in the relationship between gratitude and satisfaction with life using the macro PROCESS for SPSS through model 4 (Hayes, 2013). The data collected was analysed via the SPSS 24.0 version for Windows.

\section{Results}

Prior to designing studies, it is important to know the adequacy of the sample size required for .80 power to detect an effect (Fritz \& MacKinnon, 2007). According to Fritz and MacKinnon (2007), a sample of between 115 and 285 would be required for detecting an indirect effect among the variables. In this study, a total of 141 participants were recruited. This suggested that the sample size for the present study fell within the range of detecting an effect with .80 power.

Table 1 displays means, standard deviations, skewness and kurtosis statistics, and Pearson correlation coefficients of the study variables. As can be seen in Table 1, the mean scores for satisfaction with life and gratitude were above the midpoint of the associated scales, suggesting that participants were highly satisfied and felt grateful for their life. The mean score of perceived stress was below the midpoint of the scale, suggesting that participants perceived the situations in their life as less stressful.

Table 1 also shows Pearson product-moment correlation coefficients among the variables. When the importance of correlations between variables is assessed, it is useful to consider the effect size of correlations to have a firmer conclusion regarding the strength of a relationship between variables. According to Cohen $(1992,1988)$, a value falls within $.1 \leq r<.3$ should be considered as a small effect, a value within $.3 \leq r<.5$ as a medium effect, and a value of $r>=.5$ as a large effect. Taking this into consideration, higher scores on gratitude were significantly related with higher scores on satisfaction with life (medium effect), while higher scores on perceived stress were significantly related with lower scores on gratitude (medium effect) and satisfaction with life (large effect).

Table 1. Means, standard deviations, skewness, kurtosis, and correlations coefficients of the variables $(\mathrm{N}=141)$

\begin{tabular}{|c|c|c|c|c|c|c|c|}
\hline & 1 & 2 & 3 & Mean & $S D$ & Skewness & Kurtosis \\
\hline 1. Gratitude & - & & & 22.79 & 4.79 & 0.18 & -0.75 \\
\hline 2. Satisfaction with life & $.313^{* *}$ & - & & 21.57 & 4.01 & 0.24 & -0.96 \\
\hline 3. Perceived stress & $-.400^{* *}$ & $-.610^{* *}$ & - & 18.99 & 4.66 & -0.12 & -0.17 \\
\hline
\end{tabular}

Prior to testing indirect effect of stress in the relationship between gratitude and satisfaction with life, we first performed an array of separate regression analysis for direct effects of gratitude and stress on satisfaction with life. Results indicated that when stress as a mediator was not controlled, there were significant direct effects between gratitude and satisfaction with life $\left(B=.26, t=3.88, p<.001, r^{2}=.10\right)$, gratitude and stress $(B=-.39, t$ $\left.=-5.15, p<.001, r^{2}=.16\right)$, and stress and satisfaction with life $\left(B=-.53, t=-9.08, p<.001, r^{2}=.37\right.$ ) (see Table 2).

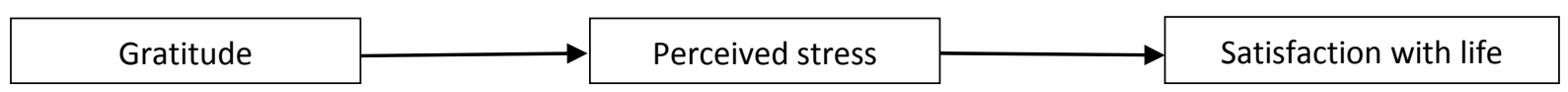

Figure 1. The model that examines the role of the perceived stress in the relationships between gratitude and satisfaction with life 
PROCESS macro for SPSS was used to perform the mediation analysis. The macro employs bootstrap procedure to generate confidence limits for the indirect effect (Hayes, 2013). The procedure has shown to be superior to Baron and Kenny's (1986) procedure and Sobel's (1982) test for testing the indirect effect. The bootstrap procedure was performed to test whether stress mediated the relationship between gratitude and satisfaction with life. To end this, we used 5.0000 bootstrap samples to estimate $95 \%$ confidence interval (CI) for mediational analysis. An indirect effect is significant when zero does not fall within the $95 \% \mathrm{CI}$. A significant result suggests that the mediator variable mediates the effect of the predictor variable on the outcome variable.

Table 2. Direct and indirect paths among the study variables

\begin{tabular}{lcccc}
\hline & $\mathrm{B}$ & $\mathrm{SE}$ & $\mathrm{t}$ & $p$ \\
\hline Gratitud $\rightarrow$ Satisfaction with life & 0.26 & 0.07 & 3.88 & $<.001$ \\
Gratitude $\rightarrow$ Perceived stress & -0.39 & 0.08 & -5.15 & $<.001$ \\
Stress $\rightarrow$ Satisfaction with life & -0.53 & 0.06 & -9.08 & $<.001$ \\
Gratitude $\rightarrow$ Perceived stress $\rightarrow$ Satisfaction with life & 0.07 & 0.06 & 1.12 & 0.266 \\
\hline
\end{tabular}

The results of mediation analysis indicated that when stress was controlled, the direct effect between gratitude and life satisfaction become insignificant $\left(B=.07, t=1.12, p=.266, r^{2}\right.$ change $\left.=.01\right)$. However, there was a significant indirect effect of stress in the relationship between gratitude and life satisfaction $(B=.19,95 \% \mathrm{CI}$ $=.10-.30$ ). Since the bootstrap confidence intervals for the indirect effect of stress did not include zero, it can be suggested that stress served as a mediator between gratitude and satisfaction with life. As the direct effect of gratitude on satisfaction with life become insignificant after inclusion of stress into the model, it can be concluded that the effect of stress was full (see Figure 1).

\section{Discussion}

This study, to the best of our knowledge, is the first empirical study to investigate the mechanism underlying the relationships between gratitude, satisfaction with life, and stress in Saudi Arabia. Particularly, this study aimed to investigate the role of perceived stress in the relationship between gratitude and satisfaction with life. The results indicated that gratitude was positively associated with satisfaction with life, while perceived stress was negatively associated with both gratitude and satisfaction with life. That is, individuals with a high degree of gratitude tend to experience lower stress and greater satisfaction with life. Many studies have been conducted to explore the relationships among gratitude and life satisfaction. The emerging findings on the relationship between gratitude and well-being in this study are in the life with previous research (e.g., Emmons \& McCullough, 2003; Kong et al., 2015; McCullough et al., 2002; Wood et al., 2008a, 2008b; 2009). The results are also compatible with previous studies on the relationship between stress and satisfaction with life (e.g., Hamarat et al., 2001; Lee et al., 2016; Simons et al., 2002; Wood et al., 2008b) and stress and gratitude (e.g., Lin, 2015; Wood et al., 2008b).

In terms of mediation analysis, the results indicated that perceived stress fully mediated the relationships between gratitude and satisfaction with life. This suggests that perceived stress, which includes the extent to which situations in one's life are perceived as stressful, may be a process that connects gratitude with greater satisfaction with life. As such, gratitude may lead to lower levels of perceived stress that further contributes to greater satisfaction with life. This has important implications for both theory and practice.

This study is the first empirical study that sheds light on the underlying mechanism between gratitude and satisfaction with life in Saudi Arabia. Most of the evidence corresponding to the relationships between gratitude, satisfaction with life, and stress (e.g., Emmons \& McCullough, 2003; Hamarat et al., 2001; McCullough et al., 2002; Simons et al., 2002; Wood et al., 2008a, 2008b, 2009) are largely based on Western contexts, raising the issue of generalizability of the findings to the other cultural contexts. The present study is important in terms of offering evidence showing that the relationship between gratitude and satisfaction with life is cross-culturally valid in a non-western country. In addition to theoretical contribution to the relevant literature, this study also has important practical implications to take into consideration. Understanding the mechanism between gratitude and satisfaction with life allows mental health practitioners to design and implement effective interventions. The results show that stress functions a significant role in decreasing gratitude's effect on satisfaction with life. Thus, by reducing the levels of stress, mental health practitioners can focus on enhancing individuals' gratitude levels to contribute greater life satisfaction when they decide on training and motivational systems aiming to foster life satisfaction.

Even though this study significantly contributes to our understanding of the mechanism underlying the 
relationships among gratitude, stress, and satisfaction with life among undergraduate students in Saudi Arabia, it is important to note a number of factors restricting generalizability of the findings. The foremost limitation is related to the cross-sectional nature of the study, limiting the ability to make causal inferences. In other words, it is impossible to conclude whether greater level of gratitude causes lower level of stress and that in turn causes greater life satisfaction or higher levels of stress cause lower sense of gratitude and that in turn causes lower life satisfaction. Collecting data via experimental and longitudinal designs would be fruitful to fully investigate the emerging mechanism. Another limitation is that the study sample was recruited using a convenient sampling procedure and included only students, with being largely females (87.2\%). Using randomly drawn sample from a target population, replication of the present findings in other samples with approximately equal numbers of males and females from diverse populations (e.g., community, adolescents, and clinical samples) would be important in generalizing the findings (Yildirim \& Belen, 2018b). The final limitation is that we only included stress variable as a mediator between the study variables. However, there are a large number of other variables affecting the relationships between the gratitude and satisfaction with life. For example, we did not consider depression and anxiety as potential mediators. It is plausible to anticipate depression and anxiety variables would serve significant mediators in the relationships between gratitude and satisfaction with life. Study using depression and anxiety as mediators between the study variables would be beneficial to comprehensively understand the emerging mechanism.

In conclusion, this study investigated the role of stress in the relationships between gratitude and satisfaction with life in an understudied Arabic population. The results indicated that there is a positive relationship between gratitude and satisfaction with life and this relationship can be addressed by the theory that being grateful for things in life and appreciating positive feelings reduce the level of perceived stress, and that in turn increases the level of satisfaction with life. This has important implications for theory and practice.

\section{References}

Abdel-Khalek, A. M. (2008). Satisfaction with life in Kuwaiti samples. Derasat Nafsiyah [Psychological Studies], 18, 121 - 135. [In Arabic]

Atanes, A. C., Andreoni, S., Hirayama, M. S., Montero-Marin, J., Barros, V. V., Ronzani, T. M. ... \& Demarzo, M. M. (2015). Mindfulness, perceived stress, and subjective well-being: a correlational study in primary care health professionals. BMC complementary and alternative medicine, 15(1), 303. https://doi.org/10.1186/s12906-015-0823-0

Baron, R. M., \& Kenny, D. A. (1986). The moderator-mediator variable distinction in social psychological research: Conceptual, strategic, and statistical considerations. Journal of personality and social psychology, 51(6), 1173-1182. https://doi.org/10.1037/0022-3514.51.6.1173

Beutel, M. E., Glaesmer, H., Wiltink, J., Marian, H., \& Brähler, E. (2010). Life satisfaction, anxiety, depression and resilience across the life span of men. The Aging Male, 13(1), 32-39. https://doi.org/10.3109/13685530903296698

Chaaya, M., Osman, H., Naassan, G., \& Mahfoud, Z. (2010). Validation of the Arabic version of the Cohen Perceived Stress Scale (PSS-10) among pregnant and postpartum women. BMC psychiatry, 10(1), 111. https://doi.org/10.1186/1471-244X-10-111

Cohen J. (1988). Statistical power analysis for the behavioral sciences (2nd ed.). Hillsdale, NJ: Erlbaum. https://doi.org/10.1016\%2Fc2013-0-10517-x

Cohen, J. (1992). A power primer. Psychological bulletin, 112(1), 155-159. https://doi.org/10.1037/0033-2909.112.1.155

Cohen, S., Kamarck, T., \& Mermelstein, R. (1983). A global measure of perceived stress. Journal of health and social behavior, 24, 385-396. https://doi.org/10.2307/2136404

Darling, C.A., McWey, L.M., Howard, S.N., \& Olmstead, S.B. (2007). College student stress: The influence of interpersonal relationships on sense of coherence. Stress and Health, 23(4), 215-229. https://doi.org/10.1002/smi.1139

Diener, E. (1984). Subjective well-being. Psychological Bulletin, 95, 542-575. https://doi.org/10.1037/0033-2909.95.3.542

Diener, E. (2000). Subjective well being: The science of happiness and a proposal for a national index. American Psychologist, 55, 34-43. https://doi.org/10.1037/0003-066X.55.1.34

Diener, E., \& Diener, M. (1995). Cross-cultural correlates of life satisfaction and self-esteem. Journal of 
Personality and Social Psychology, 68, 653 - 663. https://doi.org/10.1037/0022-3514.68.4.653

Diener, E., Emmons, R. A., Larsen, R. J., \& Griffin, S. (1985). The satisfaction with life scale. Journal of Personality Assessment, 49(1), 71-75. https://doi.org/10.1207/s15327752jpa4901_13

Diener, E., \& Lucas, R. (1999). Personality and subjective well-being. In D. Kahneman, E. Diener, \& N. Schwarz (Eds.), Well-being: The foundations of hedonic psychology (pp. 213-229). New York: Russell Sage Foundation.

Diener, E., Oishi, S., \& Lucas, R. E. (2003). Personality, culture, and subjective well-being: Emotional and cognitive evaluations of life. Annual review of psychology, 54(1), 403-425. https://doi.org/10.1146/annurev.psych.54.101601.145056

Diener, E. \& Suh, E. (1997). Measuring quality of life: Economic, social and subjective indicators. Social Indicators Research, 40, 189-216. https://doi.org/10.1023/A:1006859511756

Diener, E., Suh, E. M., Lucas, R. E., \& Smith, H. L. (1999). Subjective well-being: Three decades of progress. Psychological bulletin, 125(2), 276 - 302. https://doi.org/10.1037/0033-2909.125.2.276

Eid, M., \& Diener, E. (2004). Global judgments of subjective well-being: Situational variability and long-term stability. Social indicators research, 65(3), 245-277. https://doi.org/10.1023/B:SOCI.0000003801.89195.bc

Emmons, R. A., \& McCullough, M. E. (2003). Counting blessings versus burdens: an experimental investigation of gratitude and subjective well-being in daily life. Journal of Personality and Social Psychology, 84, 377-389. https://doi.org/10.1037/0022-3514.84.2.377

Extremera, N., Durán, A., \& Rey, L. (2009). The moderating effect of trait meta-mood and perceived stress on life satisfaction. Personality and Individual Differences, 47(2), 116-121. https://doi.org/10.1016/j.paid.2009.02.007

Fritz, M. S., \& MacKinnon, D. P. (2007). Required sample size to detect the mediated effect. Psychological science, 18(3), 233-239. https://doi.org/10.1111/j.1467-9280.2007.01882.x

Grant, N., Wardle, J., \& Steptoe, A. (2009). The relationship between life satisfaction and health behavior: a cross-cultural analysis of young adults. International journal of behavioral medicine, 16(3), 259-268. https://doi.org/10.1007/s12529-009-9032-x

Hamarat, E., Thompson, D., Zabrucky, K., Steele, D, Matheny, K., \& Aysan, F. (2001). Perceived stress and coping resource availability as predictors of life satisfaction in young, middle aged and older adults. Experimental Aging Research, 27(2), 181-196. https://doi.org/10.1080/036107301750074051

Hayes, A. F. (2013). Introduction to mediation, moderation, and conditional process analysis: A regression-based approach. New York, NY, USA: Guilford Press. https://doi.org/10.1111\%2Fjedm.12050

Kong, F., Ding, K., \& Zhao, J. (2015). The relationships among gratitude, self-esteem, social support and life satisfaction among undergraduate students. Journal of Happiness Studies, 16(2), 477-489. https://doi.org/10.1007/s10902-014-9519-2

Lambert, N. M., Fincham, F. D., Braithwaite, S. R., Graham, S. M. \& Beach, S. R. (2009). Can prayer increase gratitude? Psychology of Religion and Spirituality, 1(3), 139-149. https://doi.org/10.1037/a0016731

Lavy, S., \& Littman-Ovadia, H. (2011). All you need is love? Strengths mediate the negative associations between attachment orientations and life satisfaction. Personality and Individual Differences, 50, 1050-1055. https://doi.org/10.1016/j.paid.2011.01.023

Lazarus, R.S., \& Folkman, S. (1984). Stress, Appraisal, and Coping. Springer, New York.

Lee, J. Y., Kim, S. Y., Bae, K. Y., Kim, J. M., Shin, I. S., Yoon, J. S., \& Kim, S. W. (2018). The association of gratitude with perceived stress and burnout among male firefighters in Korea. Personality and Individual Differences, 123, 205-208. https://doi.org/10.1016/j.paid.2017.11.010

Lee, J., Kim, E., \& Wachholtz, A. (2016). The effect of perceived stress on life satisfaction: The mediating effect of self-efficacy. Chongsonyonhak yongu [Korean journal of youth studies], 23(10), 29-47. https://doi.org/10.21509/KJYS.2016.10.23.10.29

Lin, C. C. (2015). Self-esteem mediates the relationship between dispositional gratitude and well-being. Personality and Individual Differences, 85, 145-148. https://doi.org/10.1016/j.paid.2015.04.045

Lucas, R. E., Diener, E., \& Suh, E. M. (1996). Discriminant validity of subjective well-being measures. Journal of Personality and Social Psychology, 71, 616-628. https://doi.org/10.1037/0022-3514.71.3.616 
Lyubomirsky, S., Sheldon, K. M., \& Schkade, D. (2005). Pursuing happiness: The architecture of sustainable change. Review of general psychology, 9(2), 111-131. https://doi.org/10.1037/1089-2680.9.2.111

Martínez-Martí, M. L., \& Ruch, W. (2017). Character strengths predict resilience over and above positive affect, self-efficacy, optimism, social support, self-esteem, and life satisfaction. The Journal of Positive Psychology, 12(2), 110-119. https://doi.org/10.1080/17439760.2016.1163403

McCullough, M. E., Emmons, R. A., \& Tsang, J. (2002). The grateful disposition: A conceptual and empirical topography. Journal of Personality and Social Psychology, 82, 112-127. https://doi.org/10.1037/0022-3514.82.1.112

Pavot, W., \& Diener, E. (1993). Review of the satisfaction with life scale. Psychological Assessment, 5, 164-172. https://doi.org/10.1037/1040-3590.5.2.164

Praharso, N. F., Tear, M. J., \& Cruwys, T. (2017). Stressful life transitions and wellbeing: A comparison of the stress-buffering hypothesis and the social identity model of identity change. Psychiatry research, 247, 265-275. https://doi.org/10.1016/j.psychres.2016.11.039

Simons, C., Aysan, F., Thompson, D., Hamarat, E., \& Steele, D. (2002). Coping resource availability and level of perceived stress as predictors of life satisfaction in a cohort of Turkish college students. College Student Journal, 36(1), 129-141.

Sirois, F. M., \& Wood, A. M. (2017). Gratitude uniquely predicts lower depression in chronic illness populations: A longitudinal study of inflammatory bowel disease and arthritis. Health Psychology, 36(2), 122-132. https://doi.org/10.1037/hea0000436

Sobel, M. E. (1982). Asymptotic confidence intervals for indirect effects in structural equation models. In S. Leinhardt (Ed.), Sociological Methodology 1982 (pp. 290-312). Washington DC: American Sociological Association. https://doi.org/10.2307/270723

Teh, H. C., Archer, J. A., Chang, W., \& Chen, S. H. (2015). Mental well - being mediates the relationship between perceived stress and perceived health. Stress and Health, 31(1), 71-77. https://doi.org/10.1002/smi.2510

Tsigos, C., Kyrou, I., Kassi, E., \& Chrousos, G. (2016). Stress, endocrine physiology and pathophysiology. In: De Groot LJ, et al. (eds) Endotext [Internet]. South Dartmouth, MA: MDText.com, Inc.

Yildirim, M., \& Aziz, I. A. (2017). Psychometric properties of Turkish form of the Fear of Happiness Scale. The Journal of Happiness \& Well-Being, 5(2), 187-195.

Yildirim, M., Barmanpek, U., \& Farag, A.A.H. (2018a). Reliability and Validity Studies of Externality of Happiness Scale among Turkish Adults. European Scientific Journal, ESJ, 14 (14), 1-17. https://doi.org/10.19044\%2Fesj.2018.v14n14p1

Yildirim, M., \& Belen, H. (2018b). Fear of Happiness Predicts Subjective and Psychological Well-Being above the Behavioral Inhibition System (BIS) and Behavioral Activation System (BAS) Model of Personality. Journal of Positive Psychology and Wellbeing, 2(1), 92-111.

Wood, A. M., Joseph, S., \& Maltby, J. (2008). Gratitude uniquely predicts satisfaction with life: Incremental validity above the domains and facets of the five-factor model. Personality and Individual Differences, 45(1), 49-54. https://doi.org/10.1016/j.paid.2008.02.019

Wood, A. M., Joseph, S., \& Maltby, J. (2009). Gratitude predicts psychological well-being above the Big Five facets. Personality and Individual differences, 46(4), 443-447. https://doi.org/10.1016/j.paid.2008.11.012

Wood, A. M., Maltby, J., Gillett, R., Linley, P. A., \& Joseph, S. (2008). The role of gratitude in the development of social support, stress, and depression: Two longitudinal studies. Journal of Research in Personality, 42(4), 854-871. https://doi.org/10.1016/j.jrp.2007.11.003

\section{Copyrights}

Copyright for this article is retained by the author(s), with first publication rights granted to the journal.

This is an open-access article distributed under the terms and conditions of the Creative Commons Attribution license (http://creativecommons.org/licenses/by/4.0/). 